\title{
Development and Evaluation of a Workpiece Evaluation Support Mechanism for Students
}

\author{
Ryota Fukutani *, Shota Itagaki *, \\ Hideyuki Takahashi ${ }^{*}$, , Akinobu Ando ${ }^{\ddagger}$, Tetsuo Kinoshita ${ }^{*} \dagger$
}

\begin{abstract}
Japanese junior high schools teach technology education by manufacturing activities performed by the students. For instance, an activity could require the application of a specific skill like sawing. It is difficult for the students to evaluate skills objectively by themselves in the manufacturing activities. This paper proposes a mechanism for the students to facilitate the evaluation of their skills within the limited classroom hours. The mechanism evaluates angle and surface roughness of a workpiece by using the sensors of a mobile device. We implemented this mechanism and titled it 'Workpiece Evaluation Support Mechanism for Students'. Additionally, the students confirmed that the mechanism supported the realization of their task in the actual class.
\end{abstract}

Keywords: Education System, Evaluation Support, Objective Evaluation, Technology Education

\section{Introduction}

Recently, research on Information and Communication Technology (ICT) focuses on educational topics. Several solutions were published [1][2] to realize better classes, efficient school affairs, and improvement of students' information literacy. In Japan, junior high schools offer technology education classes. In these classes, the students learn handicraft skills (like sawing) by manufacturing activities (students make something for example a bookshelf). However, it is difficult for students to evaluate their own skills in the manufacturing activities due to the lack of experience. Meanwhile there is only a single teacher per a lot of students, and the teacher cannot provide individual coaching to all of the students. Hence, the students have to rely most of the time on their subjective opinion.

The skill evaluation comprises two items. The movement evaluation evaluates the student's body and tool movements. The workpiece evaluation evaluates the form and quality of the finished workpiece. The students conduct 'workpiece evaluation (inspection)' and 'manufacturing (modify)' repeatedly until the dimensional fit of workpieces in manufacturing process. The students

\footnotetext{
* Graduate School of Information Science, Tohoku University, Sendai, Japan

$\dagger$ Research Institute of Electrical Communication, Tohoku University, Sendai, Japan

* Technology Education course, Miyagi University of Education, Sendai, Japan
} 
cannot improve workpieces' quality without skill of accurate workpiece evaluation and grasping dimensional unfit even if the students improve the manufacturing skill. Considering it, both workpiece evaluation skill and manufacturing skill are important. However, there are few researches focused on workpiece evaluation in contrast with there are many researches focused on manufacturing skill in the field of education. We have focused on the workpiece evaluation skill and we have aimed to support the students in evaluating their own skills accurately.

There is the risk that students have problems using the system correctly or perceive the system as unnecessary burden. This paper proposes the Workpiece Evaluation Support Mechanism for Students (S-WESM) to encounter this problem. This paper shows that S-WESM supports the students' evaluation capabilities efficiently and objectively within an actual school class.

Moreover, the curriculum contents in technology education will be extended according to the next 'Course of Study' with new goals set toward 2021 in Japan [3]. However, in technology education, there has been a problem with reduced the number of classes. Therefore, it will be more difficult to evaluate the skills with great care for students, and the support for the efficient skill evaluation is required.

\section{Related Research and Technical Problem}

Various researches focus on skill improvement at a specific task using the objective evaluation (evaluation without dependence on judgment skill of evaluator). As we mentioned, there is a problem that it is difficult for the students to evaluate their own skills in the manufacturing activities due to the lack of experience in technology education. As the approaches to solve the problem, there are various researches. For example, a teaching material that captures the sawing movement of beginner and expert in 3D virtual space 'Second Life' [4], a movement analyze system of two students for objective comparison using KINECT sensor [5], research about movement analyze method of finger motion for skill improvement [6], and a force analyze system in pipe-cutting simulation for comparison and evaluation of user's force [7]. However, such researches do not focus on workpieces. Hence, the students still encounter difficulty that cannot improve skill about 'workpiece evaluation'.

Furthermore, several research uses familiar devices (e.g. Smartphone) as medium for objective evaluation because of their ease of operation. The word 'operate' means "handle something to accomplish the evaluation" in this paper. For example, there is an attempt to support physics education by using mobile devices for deepening the understanding of physics [8]. However, since this research does not focus on the manufacturing activity, multilateral evaluation for one workpiece is not necessary. Hence, the students cannot perceive the quality of a workpiece as good or bad. In addition, management support for teacher is not considered. For example, result management by collecting data about students' workpieces along with students' personal information in the database. This enables the teacher to identify weak spots in this class and to align his guidance to it.

As result, the existing researches are not applicable to the environment of manufacturing activities in technology education. This research gap includes following problems: (P1) Difficulty to grasp the condition of workpieces for students: A general evaluation method requires human senses (e.g. eye measurement or touch) even if student use tools. Therefore, the students have to hear a teacher to confirm their evaluation is correct or not. However, the teacher 
cannot provide individual coaching for all students, and it is difficult for the students to grasp the condition of workpieces themselves. In addition, general evaluation equipment uses various technical terms that are complex for the students to fully understand condition of workpieces.

(P2) Big burden for students to evaluate workpieces: Evaluation devices for students require an easy application that the risk of incorrect measurement is minimal and the measurements are executed fast due to limited classroom hours. In contrast to the requirements, a common workpiece evaluation device such as the surface roughness measuring machine is difficult to understand how to use it.

\section{Workpiece Evaluation Support Mechanism for Students}

We have been developing the Practice Learning Management System (PLMS) [9][10][11][12]. This system's objective is to reduce the teacher's burden and to improve junior high school students' handicraft skill. This is achieved by support of evaluation and management of students' practices. The mechanism of this system consists of workpiece and movement evaluation.

If PLMS's support mechanism of the workpiece evaluation can support the students in evaluating workpieces objectively on their own, then a self-assessment of skill by students is possible accompanied by a more efficient coaching by a teacher. However, the PLMS mechanism is developed for a teacher [11]. Specifically, the developed mechanism provides several features for the teacher to conduct different types of evaluation work. While a teacher can be trained in using the system, the students need simple functions in order to prevent confusion. Therefore, it is difficult to apply to the S-WESM.

Hence, we have aimed to solve the problems (P1) and (P2) by proposing the Workpiece Evaluation Support Mechanism for Students (S-WESM) in PLMS in the field of technology education [12]. This paper describes the realization of following solution:

(S1) Easy-to-use measurement support function: S-WESM supports easy-to-use evaluation by limiting operational complexity and eliminating unnecessary functions for the students in order to prevent incorrect operation.

(S2) Grasping condition support function: We change the evaluation information of the mechanism into comprehensive information to facilitate objective grasping of the workpiece's condition by the students.

\subsection{Learning Theory}

We describe some learning theories that were based to design the basic configuration of the SWESM. At first, Japanese students have to learn about 'Manufacturing parts, finishing, and assembly' as compulsory items on the curriculum in technology education. In the procedure of their learning, the students make a work for each student generally. Hence, there are gaps among each student's manufacturing progress inevitably. Therefore, we have aimed at realization of manufacturing activities tailored for each student's learning progress based on Bloom's Mastery Learning. Specifically, the S-WESM realizes real-time evaluation (formative assessment) and enables corresponding to individual gap of manufacturing progress.

Moreover, we referred Keller's ARCS model to give the students motivation for learning. ARCS model is focused on motivation for learning and it consists of following four components: Attention, Relevance, Confidence, and Satisfaction. We aimed at giving motivation for the students to 
customize the mechanism based on ARCS model as follows:

Attention: We expect the influence of attention for learning by using mobile device that is familiar but unusual in school class for the students.

Relevance: We aim at displaying familiar evaluation information for the students by eliminating difficult information for students.

Confidence: Real-time feedback design aims at improvement of the students' confidence.

Satisfaction: We expect to keep satisfaction in term of keeping fairness by objective evaluation based on sensors.

Additionally, S-WESM enables the teacher to browse the students' evaluated data in real-time for improving authenticity of teacher's advises.

\subsection{Basic Configuration}

We describe overview, design and implementation of the S-WESM in comparison to the Workpiece Evaluation Support Mechanism for Teacher [11] (T-WESM) because the S-WESM originates from the T-WESM.

Figure 1 shows the position of the S-WESM in the PLMS. The S-WESM serves as a mechanism for the students' Workpiece Evaluation Support Mechanism. Eventually, this system aims at "revise and improve the students' own skill", "effective individual teaching and mutual evaluation", "efficient learning evaluation", and so on. The S-WESM supports the measurement, analysis of state, integration and management of the result data about the workpiece. The S-WESM has the Angle Evaluation Function for Students and the Surface Roughness Evaluation Function for Students, because they are easy to correlate with sawing and planing. These are typically required skills in technology education. Both functions retrieve student information (grade, class, number, and name) which is stored in the database as a common design in PLMS, and display it on the mobile device. Furthermore, both functions upload the evaluation information (e.g. angle data, surface roughness data) to the database on the Web server with related student information. Moreover, the teacher can centralized manage the data by accessing a dedicated Web page in a browser. The functions are also designed for Android OS applications.

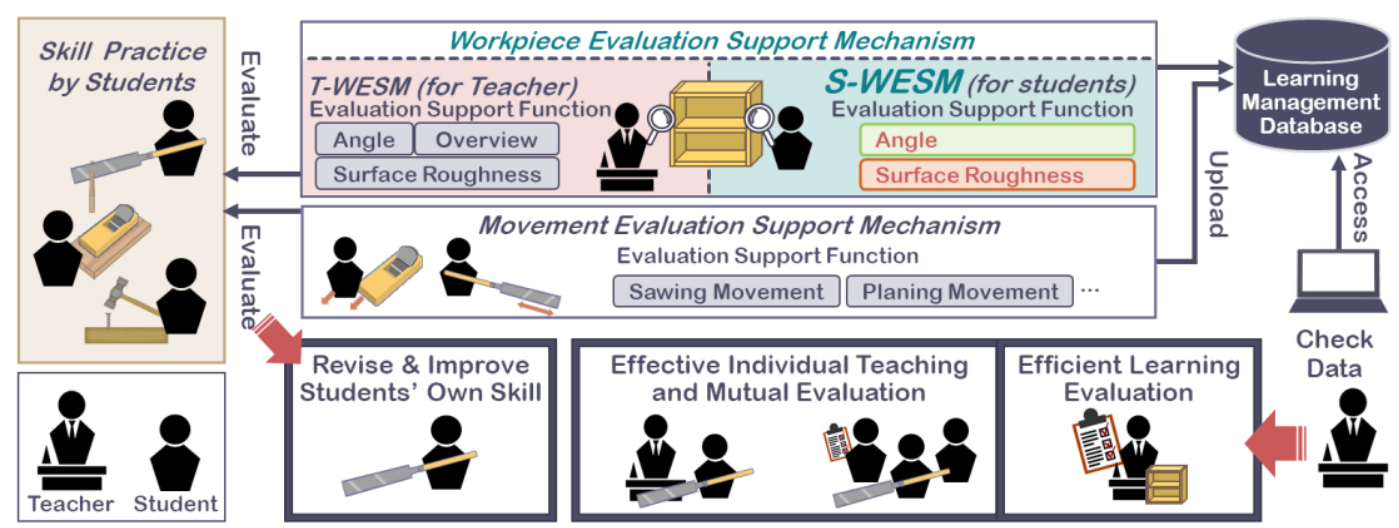

Figure 1: Position of the S-WESM in the PLMS

Figure 2(a) shows an example of the Angle Evaluation Function of T-WESM and Figure 2(b) shows an example of the Surface Roughness Evaluation Function of T-WESM. These functions for teacher are difficult to apply to the S-WESM for students. There are three differences between the T-WESM and the S-WESM regarding the target group. Firstly, the number of users per school 
is one teacher in the T-WESM but 100 students (case of general one class) in the S-WESM. Secondly, the use case is for teacher after school but for students during class. Thirdly, the TWESM evaluates workpieces of the entire grade, while the S-WESM focuses on one workpiece. Considering those three differences, following requirements are crucial for the S-WESM: 1. Easy approach for operations, 2. Clear and comprehensive evaluation information, and 3. Simple evaluation procedure. The following section describes each developed function meeting above requirements.

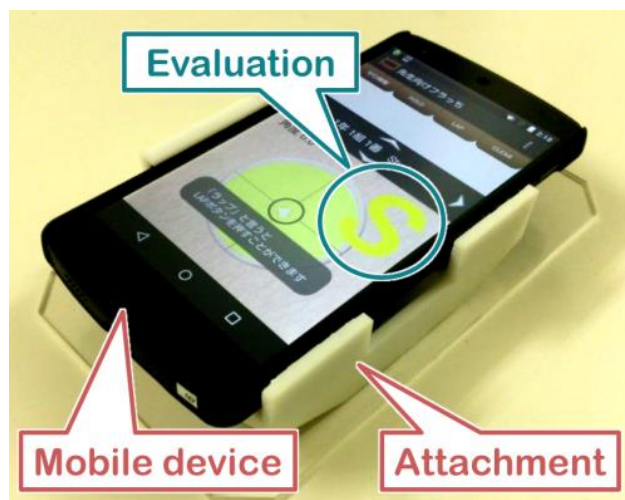

(a) Angle Evaluation Function

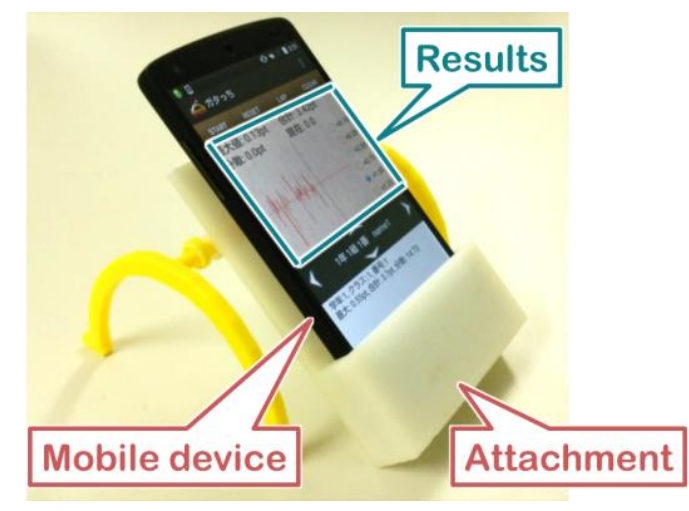

(b) Surface Roughness Evaluation Function Figure 2: Example of the T-WESM

\subsection{Angle Evaluation Function for Students}

Angle Evaluation Function for Students of the S-WESM enables objective evaluation by quantifying the angle of the workpiece (face) using a gravity sensor built into the mobile device. In action, students check the angle of workpieces with the mobile device instead of using a steel square (a kind of tool in conventional methods). The function ensures a more accurate angle measurement with use of the angle measurement attachment that prevents the influence of the shape of the mobile device. Students can measure the angle by the contact between attachment and surface of a workpiece. Figure 3(a) shows an example of this function and Figure 3(b) shows the results after evaluation. This S-WESM's function has the following differences compared to the T-WESM's function: (A) Objective evaluation support, (B) Simplification of configuration, and $(\mathrm{C})$ Automatic upload.

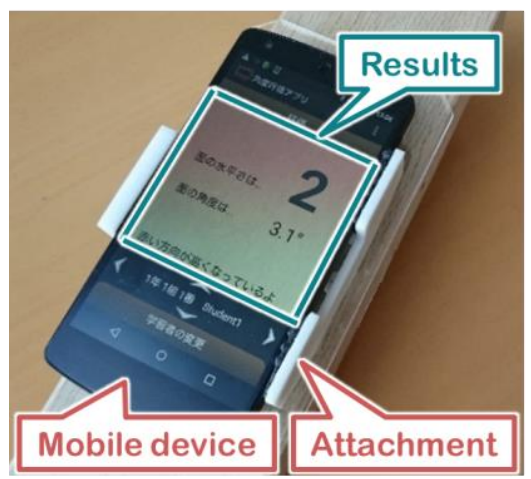

(a) Appearance

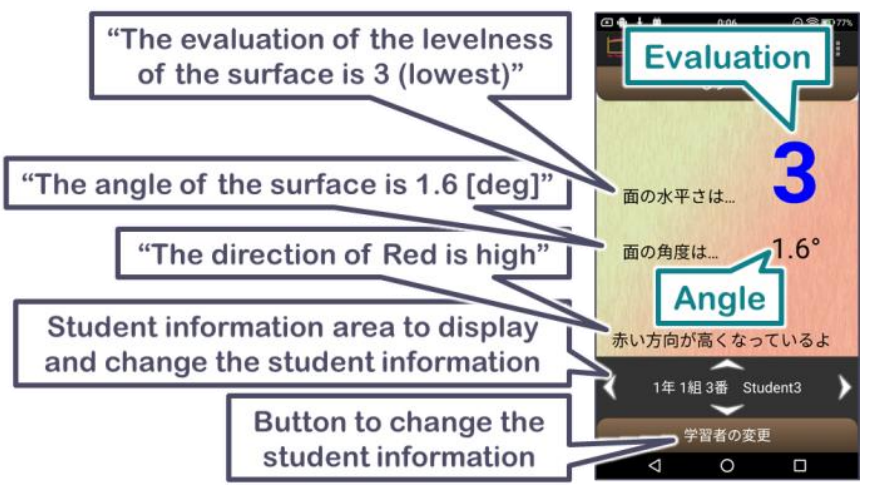

(b) Screen of results

Figure 3: Example of the Angle Evaluation Function of the S-WESM 
The function visualizes the direction of the slope in color gradation, and the 3-scale evaluation of the angle in numbers $\{1-3\}$. The result is an objective evaluation (A). It supports the students' objective grasping of the workpiece's condition. The T-WESM visualizes the slope using a level (a kind of tool) as shown in Figure 2(a). However, the students need a simple representation to easily understand the evaluation result because of a risk that they do not know the level and they cannot understand the meaning of it. Therefore, we introduced a color representation method of the slope as shown in Figure 3(b).

The students need simple functions because of the risk that the students become confused. As for (B) Simplification of configuration, we reduced the available functions only to the essential ones as shown in Figure 4. This function's amount of steps is reduced by about $54 \%$ compared to the T-WESM's function as shown in Figure 4(a). Additionally, we aimed at minimization of potential mistakes during students operation by highlighting only the necessary parts for the students. Moreover, we reduced the evaluation information from 9 types to 7 types (22\%) as shown in Figure 4(b).

\begin{tabular}{|c|c|c|}
\hline \multirow{2}{*}{ Step No. } & \multicolumn{2}{|c|}{$\begin{array}{c}\text { Angle Evaluation Function } \\
\end{array}$} \\
\hline & T-WESM & S-WESM \\
\hline & Set a measure angle & Choose a grade \\
\hline & Push the OK button & Choose a class \\
\hline & Set standards & Choose a student number \\
\hline & Push the OK button & Touch this function with a workpiece \\
\hline & Choose a grade & Push Evaluation button \\
\hline & Choose a class & Make sure the evaluation information \\
\hline & Choose a student number & -1 \\
\hline & Touch this function with a workpiece & - \\
\hline & Wait 1 second & \\
\hline & Push the LAP button & \\
\hline 11 & Push the Menu button & by $\sim 54 \%$ \\
\hline 12 & Push the Export in the menu & - \\
\hline 13 & Make sure the evaluation information & - \\
\hline
\end{tabular}

(a) Evaluation steps

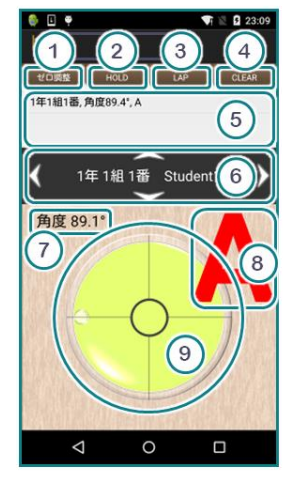

T-WESM

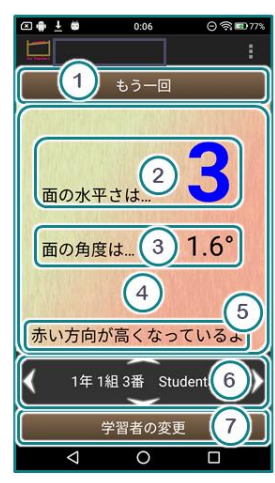

S-WESM

(b) Evaluation information

Figure 4: Differences between T-WESM and S-WESM of the Angle Evaluation Function

As described in Section 3.2, the teacher needs to evaluate many works, and they do not need to export the evaluated data each time. Hence, the T-WESM's function includes a step to record data temporarily and to export all data at once. The S-WESM's function uploads the evaluation data automatically each time after the students evaluate an angle as for (C) Automatic upload. Afterwards, the teacher can browse states of the students' work.

\subsection{Surface Roughness Evaluation Function for Students}

Surface Roughness Evaluation Function for Students of S-WESM evaluates the mobile device's vibration as the surface roughness. Normally, students inspect the surface roughness of workpieces by observation and touch using their sense and the square. The students inspect it by using a mobile device in proposed method. This function uses the surface roughness measuring attachment together with the Angle Evaluation Function. Figure 5(a) shows an example of this function. Figure 5(b) shows a screen after evaluation. This S-WESM's function has the following differences compared to the T-WESM's function: (A) Objective evaluation support, (B) Simplification of configuration, (C) Automatic upload, and (D) Improved attachment. 
This function expresses the 3-scale evaluation and translates the surface roughness into points to achieve (A) Objective evaluation support. The function visualizes the 3-scale evaluation of the surface roughness in numbers $\{1-3\}$. Although the T-WESM's function displays some objective data, they are difficult to understand for students (e.g. variance). Therefore, we eliminated all difficult terms and introduced the 'surface roughness point' for students to easily understand objective data. A value of the surface roughness points is equivalent to a variance of gyro sensor's values built into the device that are acquired during 3 seconds. Furthermore, the T-WESM requires the teacher to count the evaluation time by pressing a button 'Start/Finish'. However, it is necessary to pull the workpiece slowly at the same time. Those operations in parallel are difficult for students. Hence, we implement automatic evaluation in the S-WESM. This operation is only pulling the workpiece during the countdown by sound and texts on the screen. Those measures are supporting easier objective grasping of the condition of the workpiece for the students.

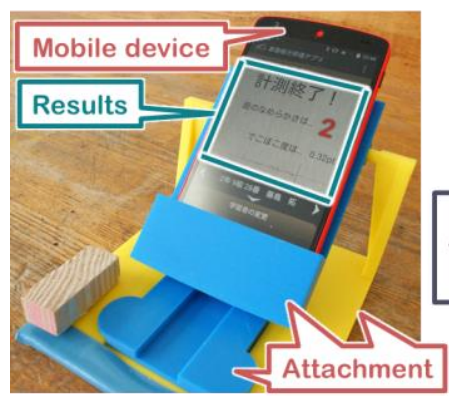

(a) Appearance

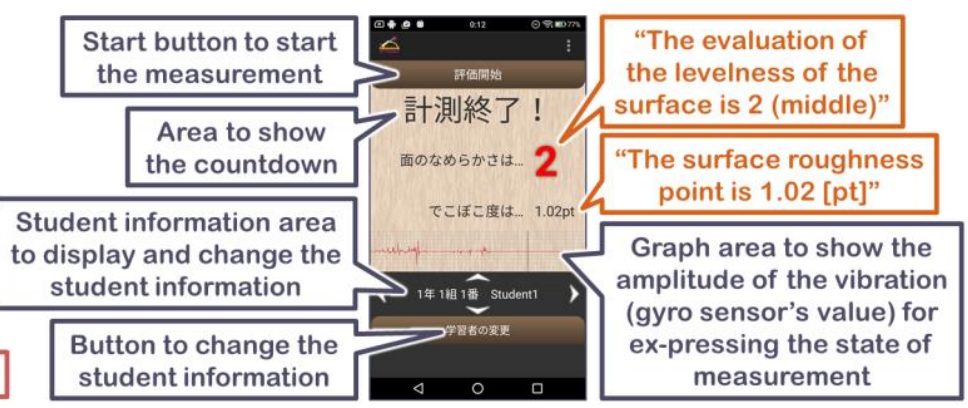

(b) Screen of results

Figure 5: Example of the Surface Roughness Evaluation Function of the S-WESM

We achieve (B) Simplification of configuration and (C) Automatic upload with the new Angle Evaluation Function. We reduced the evaluation steps by about 36\% as shown in Figure 6(a), and reduced the evaluation information from 12 types to 7 types (42\%) as shown in Figure 6 (b). More-over, we highlighted the necessary parts for students as shown in Figure 5(b).

\begin{tabular}{|c|c|c|}
\hline \multirow{2}{*}{ Step No. } & \multicolumn{2}{|c|}{ Surface Roughness Evaluation Function } \\
\hline & T-WESM & S-WESM \\
\hline 1 & Choose a grade & Choose a grade \\
\hline 2 & Choose a class & Choose a chass \\
\hline 3 & Choose a student number & Choose a student number \\
\hline 4 & Set a workpiece under the attachment & Set a workpiece in the slider \\
\hline 5 & Push the Start button & Push the Start button \\
\hline 6 & Pull the workpiece during 3 seconds & Pull the workpiece during the countdown \\
\hline 7. & Push the Stop button & Make sure the evaluation information \\
\hline 8 & Push the LAP buttion & - \\
\hline 9 & Push the Menu buttion & \\
\hline 10 & Push the Export in the menu & \\
\hline 11 & Make sure the evaluation information & \\
\hline
\end{tabular}

(a) Evaluation steps

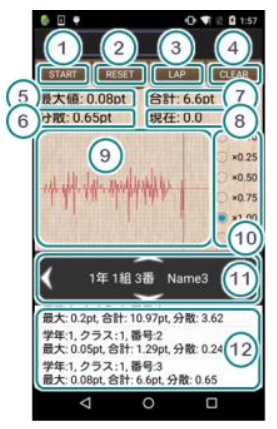

T-WESM

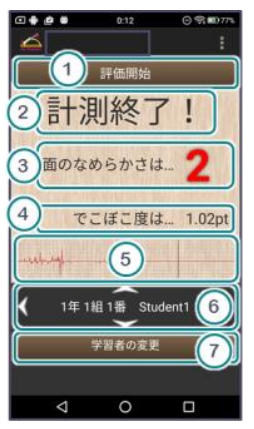

S-WESM

(b) Evaluation information

Figure 6: Differences between T-WESM and S-WESM of the Surface Roughness Evaluation Function

The developed attachment in the previous study (T-WESM's attachment) had some problems to improve. We modified the attachment made in 3D printer from Figure 7(a) to Figure 7(b) to achieve (D) Improved attachment. Firstly, we added the base plate to prevent unnecessary vibrations. T-WESM's attachment required the user to pull the workpiece on the smoothest surface. Secondly, we added a slider structure that manages the pulling range because of the previous 
difficulty to stop pulling at edge of a workpiece. Thirdly, we reinforced the structure because it showed risk of breaking.

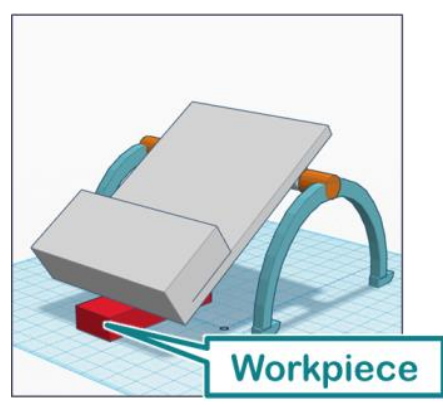

(a) Previous attachment

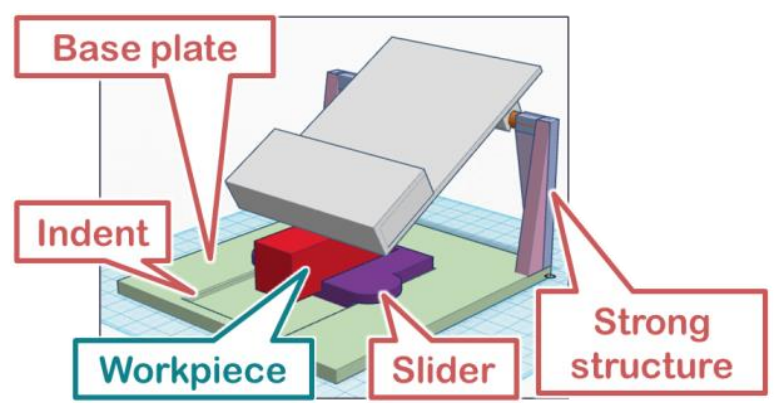

(b) Improved attachment

Figure 7: Difference of the attachment depends on the improvement

\section{Evaluation}

This section verifies the S-WESM's convenience and easiness for students of grasping to evaluate workpieces through two usability verifying experiments.

\subsection{Comparison between T-WESM and S-WESM}

Although UI and procedure of T-WESM and S-WESM work in the different way, T-WESM works in the same way with S-WESM in terms of workpiece evaluation. Hence, the students can also use T-WESM if the students were taught how to use. Therefore, we have to verify whether we could develop efficient mechanism for the students or not. This experiment compares the TWESM with the S-WESM using the evaluation time students require to evaluate and the interview. Test subjects are 18 junior high school students in first grade $(12,13$ years old) and objects for evaluation are 6 workpieces ( 3 pieces for angle evaluation and 3 pieces for surface roughness evaluation).

Experimental procedure is as follows and conduct using both of the Angle Evaluation Function and the Surface Roughness Function:

1. The author describes overview of this experiment and how to use each function.

2. Students evaluate 3 workpieces using T-WESM.

3. Students evaluate same workpieces using S-WESM.

4. Technology education teacher interviews students.

We measured time at step 2 and 3. Meanwhile, we performed this experiment only for comparison of operational time. Hence, we gave an explanation such as "your goal is to finish the operation of evaluating workpieces" to students at step 1. Although we did not prepare a group that replaced step 2 and 3, the students' goal is not to answer the evaluation result of workpiece, so there is no influence by order of step 2 and 3.

Figure 8 shows the average evaluation time. The p-values in Figure 8 are calculated by the paired samples t-test, and Table 1 shows one of statistical analysis data. We reduced the evaluation time of the S-WESM's function by about 31 seconds in the Angle Evaluation Function (Figure 8(a)), and about 20 seconds in the Surface Roughness Evaluation Function (Figure 8(b)). Thus, the 
evaluation time by the S-WESM is shorter than the evaluation time by the T-WESM. Hence, we could solve the (P2) Big burden for students to evaluate workpieces.

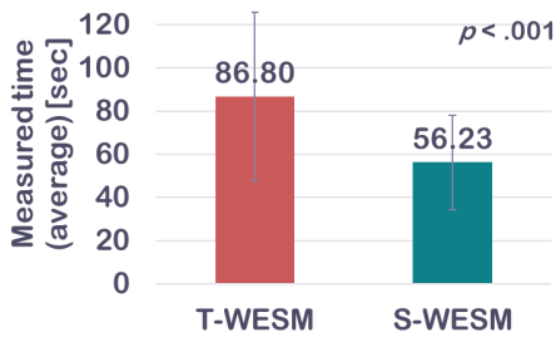

(a) Angle Evaluation Function

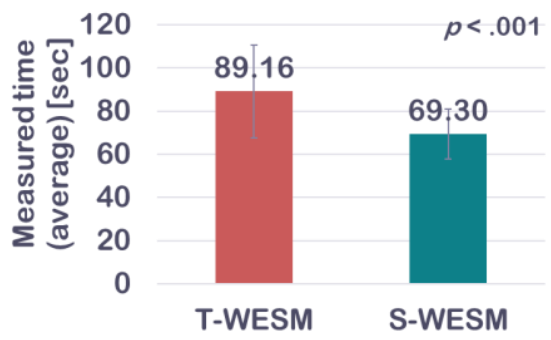

(b) Surface Roughness Evaluation Function

Figure 8: Evaluation time by students used T-WESM and S-WESM

Table 1: Paired samples t-test

\begin{tabular}{|c|c|c|c|c|c|c|c|c|}
\hline & \multirow[t]{2}{*}{ Mean } & \multirow[t]{2}{*}{$\begin{array}{l}\text { Std. } \\
\text { Devia- } \\
\text { tion }\end{array}$} & \multirow[t]{2}{*}{$\begin{array}{l}\text { Std. } \\
\text { Error } \\
\text { Mean }\end{array}$} & \multicolumn{2}{|c|}{$\begin{array}{l}95 \% \text { Confidence } \\
\text { Interval of } \\
\text { the Difference }\end{array}$} & \multirow[t]{2}{*}{$\mathrm{t}$} & \multirow[t]{2}{*}{$\mathrm{df}$} & \multirow[t]{2}{*}{$\begin{array}{l}\text { Sig. } \\
(2- \\
\text { tailed) }\end{array}$} \\
\hline & & & & Lower & Upper & & & \\
\hline $\begin{array}{c}\text { S-WEST } \\
\& \text { T-WEST } \\
\text { (Angle Evalua- } \\
\text { tion Function) }\end{array}$ & 30.57 & 26.41 & 6.24 & 17.44 & 43.70 & 4.91 & 17 & .000 \\
\hline $\begin{array}{c}\text { S-WEST } \\
\text { \& T-WEST } \\
\text { (Surface Rough- } \\
\text { ness Evaluation } \\
\text { Function) }\end{array}$ & 19.87 & 17.04 & 4.02 & 11.40 & 28.34 & 4.95 & 17 & .000 \\
\hline
\end{tabular}

Additionally, we conducted a semi-structured interview to analyze the students' impression. The interviewer is their teacher. The question items have two selection options and the teacher asked the answers' reasons. The questions are as follows: "Q $\mathrm{Q}$. Which application did you feel faster to evaluate?", "Q2. With which application did you feel that you made more mistakes during the evaluation process?", "Q3. Which application do you want to use again?", and "Q4. Which attachment was easier to use for evaluating? (Only the Surface Roughness Evaluation Function)".

Figure 9 shows the result of the interview about the Angle Evaluation Function (Figure 9(a)) and the Surface Roughness Evaluation Function (Figure 9(b)). Over 83\% students answered that the S-WESM is superior to the T-WESM in all questions. The most replied reasons of the answers are as follows: "A1. Fewer evaluation steps", "A2. Bigger contents and characters, and minimum buttons", "A3. Easy and clear operations", and "A4. The slider protects a workpiece of falling down". In addition, almost all students wrote positive comments like "Understandable". As result, we solved the (P1) Difficulty to grasp the condition of workpieces for students.

On the other hand, the students who answered that the T-WESM's Angle Evaluation Function is better than the S-WESM's one commented such as "The level is easier than the gradation to understand the direction of the slope". Moreover, the students who preferred the T-WESM's Surface Roughness Evaluation Function commented such as "It took time to insert the workpiece into slider". However, the students who preferred the T-WESM's function were fewer than $12 \%$ 
in all the questions, and they could operate both the S-WESM's functions and the T-WESM's functions (even though developed for teacher) efficiently according to our observation. Hence, we confirmed that the S-WESM's function is easy to use for evaluating workpieces for students in general. Additionally, we received ideas from the students to improve the system such as "Enhance smartphone connection with slider to prevent slipping", and we are going to refer to them. To wrap up, we could confirm that the students cannot use T-WESM easily so developing student version mechanism was required, and we could develop it at low cost.

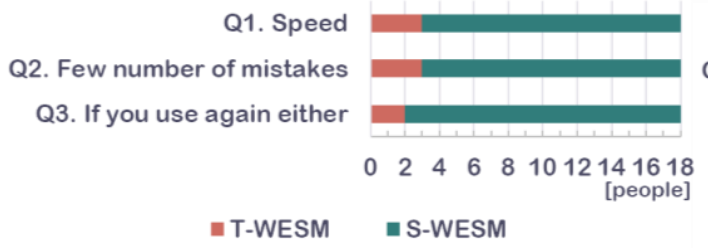

(a) Angle Evaluation Function

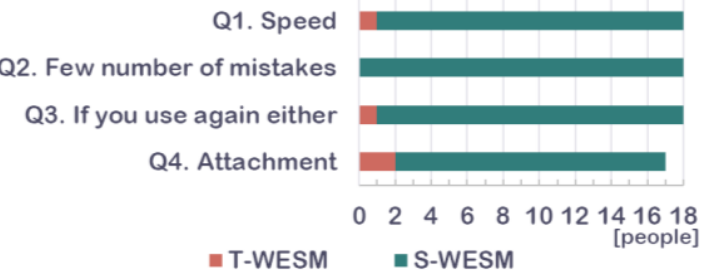

(b) Surface Roughness Evaluation Function

Figure 9: Interview results after students used the T-WESM and the S-WESM

\subsection{Verification of S-WESM in an actual class}

We performed an experiment to confirm the effectiveness the S-WESM in the actual class by comparing the proposed method (with the S-WESM) with the conventional method (using the square). The objective of the class is to master inspection skill using the square. The students experience the proposed method as a suitable assessment tool of their inspection skill. We handed out 4-point scale questionnaires to students after the class and the interview to the technology education teacher. Test subjects are 324 junior high school students in second grade (13-14 years old). Test objects for the evaluation are workpieces for both angle evaluation and surface roughness evaluation.

We explain about the class. The teaching content is 'Inspection of workpieces'. The students had learned sawing and planing until the previous class, and the workpieces that they evaluate at this class are pieces that they manufactured at the learning. Moreover, the students compare the evaluated result on their own with one by S-WESM (as correct answer data). Meanwhile, the students had learned how to evaluate in the conventional method by beforehand distributed print until the class.

Experimental procedure is as follows:

1. Technology education teacher describes how to use each function using a video tutorial.

2. Students evaluate a workpiece using the conventional method.

3. Students evaluate a same workpiece using the proposed method, and compare with evaluation by step 2 .

4. Change a workpiece and repeat step 2 and step 3.

The number of workpieces was dependent on the evaluation time given in class (two workpieces were manufactured in saw-processed and plane-processed by students themselves).

Table 2 shows the question items of the questionnaires (* = Inverted item). Q1-Q3 are items to confirm the ease of grasping objective data by the S-WESM's representation. Q4-Q8 are items to verify the proposed method's usability in contrast to the conventional method. Q9-Q11 are items to confirm the students' capability to proceed on their own. Q12 and Q13 are items to 
confirm the students' motivation about using the proposed method. Students answered the questionnaire using a 4-point Likert-scale (1. Disagree, 2. Somewhat disagree, 3. Somewhat agree, and 4. Agree). Moreover, we provided a comment section.

Table 2: Question items

\begin{tabular}{|l|}
\hline - When I evaluated the workpiece's angle or surface roughness using the smartphone... \\
\hline Q1. To be evaluated in the number "1,2,3" was easy to understand. \\
\hline Q2. Numerically expression as "Degree" and "Roughness point" was easy to understand. \\
\hline Q3. To be expressed in the graph or the color was easy to understand. \\
\hline - Compared to the evaluation using the square, the evaluation using the smartphone is... \\
\hline Q4. Simple, Q5. Tiring*, Q6. Like \\
\hline - About using smartphone to evaluate the workpiece's angle or surface roughness... \\
\hline Q7. It is convenient that can be evaluate automatically, Q8. Overall, easy to use \\
\hline - After this, if I use the smartphone... \\
\hline Q9. I can fix my inspection method by myself when I inspect workpieces using a square. \\
\hline Q10. I can determine how much a workpiece's surface is rough by myself after I saw it. \\
\hline Q11. I can determine how much a workpiece's face slopes by myself after I plain it. \\
\hline - I want to use the smartphone for... \\
\hline Q12. the training of inspection again. \\
\hline Q13. the inspection in the actual manufacturing. \\
\hline
\end{tabular}

Figure 10 shows the results. The results of Q1-Q3 indicate over 90\% students showed positive responses, and it means that the features to realize an easily grasp of objective data worked in actual class. The results of Q4-Q8 indicate over 87\% students showed positive responses, which means that the S-WESM is superior for students in term of usability compared to the conventional method. The results of Q9-Q11 indicate over 86\% students showed positive responses, which means that the students can evaluate workpieces without any action of the teacher using the proposed method. Moreover, the results of Q12 and Q13 indicate over 79\% students showed positive responses, which means that most students were satisfied and want to use the proposed method again.

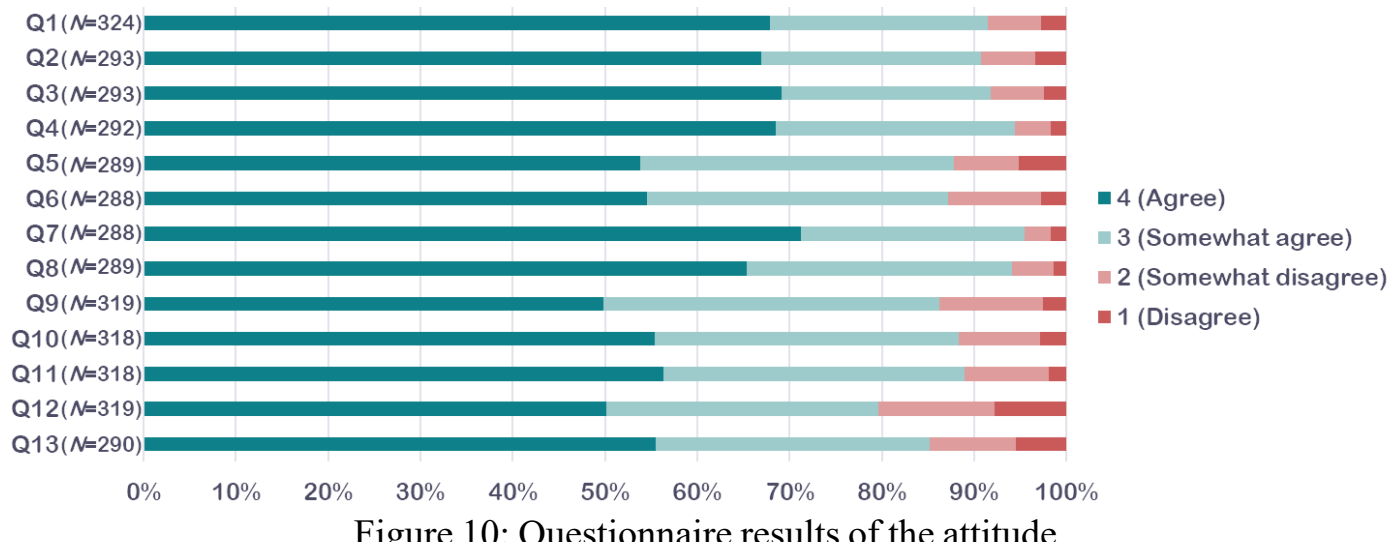

The comment section provided a few negative responses such as "Risk of breaking" or "Teacher's advice is better". Therefore, it was required to improve the attachment and it is important that the teacher and the S-WESM co-exist in class. There were many positive answers such as "the proposed method is easy to understand/easy to use/convenient". Furthermore, there was a comment "It was a comforting environment for the students feeling that there is one teacher for each students". Hence, the S-WESM supports the teacher's conduct. In other words, we could 
achieve the support function for junior high school students to evaluate workpieces objectively in the current technology education.

Additionally, we interviewed the teacher about merit of S-WESM. Relating to teacher's activity, there was a comment such as "Checking the class individually was easy because I could browse the evaluation data of student and pay attention to the students who are not good at inspection". In terms of students' attitude, there were comments such as "Students inspected their own compared to annual class although they always cannot care much for inspection" and "The number of students who act with other students were bigger than annual class". Moreover, there was a comment "If S-WESM could be developed more one-size-fits-all design and introduced into real manufacturing process, I expect that the quality of the students' workpieces improves" and we are going to refer to them.

\section{Conclusion}

This paper proposes a Workpiece Evaluation Support Mechanism for Students. The students can use our proposed mechanism to evaluate workpieces objectively on their own. We implemented a mechanism and confirmed that the mechanism is easy to use for students. In addition, it was suggested the student can perform workpiece evaluation without teacher's guidance. For our future works, we will confirm the influence on manufacturing works and the quality of workpieces by S-WESM when we apply it into actual manufacturing process. Therefore, an attachment extension and to gather more evaluation terms besides angle and surface roughness are planned. Additionally, we will implement an advanced Mutual Evaluation.

\section{Acknowledgement}

We would like to thank Ayumi Ota (Saiwaicho junior high school) for cooperating with experiments. This work was partially supported by the Student Project of Graduate School of Information Sciences, Tohoku University.

\section{References}

[1] Y. Ono and Fu Suro, "Speaking Practice Support System for Japanese Learners of English and Investigation of Learning Strategies and Patterns through Learning Log Data" Journal of Information Engineering Express, Vol.2, No.4, pp.31-40, 2016.

[2] S. Usui, K. Sato and T. Horita, "Development and Evaluation Experiment of Display Media using VR for Art Appreciation Learning," Proc. of the 6th International Congress on Advanced Applied Informatics (AAI 2017), pp.694-697, July 2017.

[3] Ministry of Education, Culture, Sports, Science and Technology Japan, http://www.mext.go.jp/a_menu/shotou/new-cs/1384661.htm (in Japanese) (accessed 201709-21).

[4] A. Ando and Y. Sumikawa, "Development and Function Evaluation of Teaching Materials for 'sawing' Observation by using Motion Capture and the Virtual World," Japan Society for Educational Technology, Vol.36, No.2, pp.103-110, Oct. 2012 (in Japanese). 
[5] S. Kurebayashi, K. Kobayashi and S. Kanemune, "Development of a basic motion analysis system using a sensor KINECT," IPSJ SIG Technical Report, Vol.2013-CE-118, No.20, pp.17, Feb. 2013 (in Japanese).

[6] R. Aoki, M. Omura and S. Kurebayashi, "A Proposal of Skill Training Using a Basic Motion Capture System,” Informational education symposium (SSS2014), No.2, pp.185-188, Aug. 2014 (in Japanese).

[7] N. Akshay, S. Deepu, E. S. Rahul, R. Ranjith, J. Jose, R. Unnikrishnan and RR. Bhavani, "Design and evaluation of a Haptic simulator for vocational skill Training and Assessment," Proc. of the 39th Annual Conference of the IEEE Industrial Electronics Society (IECON 2013), pp.6108-6113, Nov. 2013.

[8] M. Oprea and C. Miron, "Mobile Phones in the Modern Teaching of Physics," Journal of the Romanian Reports in Physics, Vol.66, No.4, pp.1236-1252, Jan. 2014.

[9] A. Ando, T. Takaku, S. Itagaki, T. Torii, H. Takeno and D. Davis, "Development of a Skill Learning System using Sensors in a Smart Phone for Vocational Education," Proc. of the 5th International Conference on Computer Supported Education (CSEDU 2013), pp.683-687, Oct. 2013.

[10] S. Itagaki, A. Ando, T. Takaku, H. Takeno and T. Torii, "Development of a Skill Practice Management System (PMS) for Learning Japanese Traditional Craft Tools by Using Smartphones," Proc. of the World Conference on Educational Multimedia, Hypermedia and Telecommunications (EDMEDIA2014), pp.1001-1009, July 2014.

[11] R. Fukutani, S. Itagaki, H. Takahashi, A. Ando and T. Kinoshita, "Evaluation Support Mechanism of Wooden Works for Practice Learning Management System of Technology Education," Trans. of Consumer Device and Systems (CDS), pp.51-63, May 2017 (in Japanese).

[12] R. Fukutani, S. Itagaki, H. Takahashi, A. Ando and T. Kinoshita, "Development of a Workpiece Evaluation Support Mechanism for Students," Proc. of the 6th International Congress on Advanced Applied Informatics (AAI 2017), pp.740-745, July 2017. 